\title{
Aquaporin 2 Expression in Human Fetal Kidney Development
}

\author{
Gergő Ráduly, Zsuzsánna Pap*, Loránd Dénes, Annamária Szántó, Zoltán Pávai \\ Department of Anatomy and Embryology, University of Medicine and Pharmacy of Tîrgu Mureș
}

\begin{abstract}
Introduction: The metanephrogenic zone, renal cortex and renal pyramids develop into their final form by week 13. The metanephric kidney produces large quantities of diluted urine in order to maintain volumes of amniotic fluid. Aquaporins are transmembrane protein channels that enable water transport through biological membranes. Aquaporin 2 (AQP2) is a water channel found in the supranuclear region and apical area of the cell membrane of the kidneys collecting tubule cells. Its main function is reabsorption of water through vasopressin stimulation. Materials and methods: Immunohistochemistry was used to study fetal renal tissue of 34 post-mortem fetuses of 9 weeks to 24 weeks gestational age. Results: AQP2 expression is present in connecting tubules and collecting tubules during the targeted time period. From week 9 to 12, the expression is cytoplasmic. From week 13 to 20 the enhancement of expression in the apical cell membrane occurs with the advancement of fetal age. At the end of the studied period, from week 21 to 24, both cytoplasmic and apical expression were observed. In animal studies AQP2 expression has an increasing trend during development. In contradiction with these results, other authors described low AQP2 levels in the human fetal kidney. Conclusions: This study helps to understand the amniotic fluid's homeostasis during pregnancy. In the beginning of the fetal period AQP2 protein is present in the cytoplasm of epithelial cells of the collecting duct and distal connecting duct. During the fetal period, AQP2 expression in collecting ducts becomes more enhanced in the apical membrane of the cells.
\end{abstract}

Keywords: aquaporin 2, fetal kidney, human, development

Received 20 March 2018 / Accepted 14 June 2018

\section{Introduction}

The kidney develops from two sources: the metanephric mesoderm and the ureteric bud. During week 5 of development the metanephric mesoderm develops from the intermediate mesoderm, and the ureteric bud develops from the mesonephric duct $[1,2,3,4]$. At the beginning of week 6 the ureteric bud gives rise to the primitive renal pelvis. This divides dichotomically in the metanephric tissue, and by the end of the week the primitive renal lobe is recognizable $[1,2,4]$. During week 7 the nephron develops from the metanephric mesoderm that surrounds collecting tubules. Initially an "S" shaped tubule is formed $[1,3]$. During week 8 the first generations of nephrons are already present, and by the end of the week typical histology of each segment is visible. The first glomeruli appear in the ninth week. During week 12 the renal sinus, minor and major calyces, as well as the papillae display their final form. During week 13 the metanephrogenic zone, renal cortex and renal pyramids develop into their final form [1, 3]. The fetal metanephric kidney produces large quantities of diluted urine in order to maintain amniotic fluid [5].

Aquaporins are transmembrane protein channels that enable water transport through biological membranes. These are made up by approximately 270 amino acid residues, and are organized into the membrane as homotetrameres; each monomer has an independent water pore. There are13 aquaporins (aquaporin 0 to 12) subdivided into three subfamilies: aquaporin subfamily

* Correspondence to: Zsuzsánna Pap

E-mail: papzsuzsa@yahoo.com
(AQP0, 1,2,4,5,6,8,10), aquaglyceroporin subfamily (AQP $3,7,9)$ and superaquaporin subfamily $[5,6,7,8,9]$.

Aquaporins are found in many organs of mammals, and play the following roles: prevention of dehydration, water secretion, transport of water through the blood-brain barrier, conditioning of sensory systems, lipid metabolism, cell motility and metastasis, and cell adhesion. Most types of aquaporins are expressed in the kidneys, which is responsible for water homeostasis of the body: AQP1, AQP2, AQP3, AQP4, AQP6, AQP7, AQP8 and AQP11 $[6,8]$.

Aquaporin 2 (AQP2) is a water channel is mostly localized in the collecting tubules of the kidney, but it is also found in connecting tubules. Its main function is vasopressin mediated reabsorption of water.

AQP2 is stored in intracellular vesicles. Upon stimulation it moves into the cell membrane where it functions as a water channel. The most important stimulation factor is vasopressin, which translocates AQP2 through adenyl cyclase (AC), elevation of cAMP levels and activation of the protein kinase A (PKA) intracellular signaling pathway $[7,9]$.

The aim of this study was to examine the expression pattern of AQP2 in human developing fetal kidney.

\section{Materials and methods}

34 post mortem fetuses of 9 to 24 weeks from the Laboratory of Pathology, Emergency County Hospital of Tîrgu Mureş, Romania were used for the study. The study was approved by the Ethical Committee of the University of Medicine and Pharmacy of Tîrgu Mureş, Romania. Signed consent were obtained from the mothers for the use of the 
post mortem fetuses for research purposes. The age of the fetuses was established based on the crown-rump length, and together with their gender, it was used to classify the fetuses into the groups shown in the table below (Table I).

The dissection of the fetuses was performed and kidney samples were collected, fixed in $4 \%$ formaldehyde overnight, and then embedded in paraffin. All of the specimens were provided from fetuses without kidney malformations. The $5 \mu \mathrm{m}$ thick sections obtained from the paraffin embedded resection tissue specimens were routinely dewaxed and rehydrated. The endogenous peroxidase was blocked by a 10 minutes $3 \% \mathrm{H}_{2} \mathrm{O}_{2}$ bath. Antigen retrieval was performed by pressurized steam cooking for 20 minutes in citrate solution ( $\mathrm{ph}=6)$. Primary AQP2 rabbit polyclonal antibody was used (ThermoFisher, Rockford, USA), 1:200, 50 minutes. Secondary Envision Flex/HRP (DAKO, Glostrup, Denmark) for 30 minutes was used for signal amplification and $\mathrm{DAB}$ chromogen was used for detecting the primary antibodies. This was followed by Hematoxylin counterstaining. The histologic aspect of the specimens was not modified. In order to quantify and compare the AQP2 immunoexpression, the location and intensity of AQP2 expression was followed. If there was no staining it was considered it negative.

Table I. Grouping of the fetuses according to age

\begin{tabular}{lccc}
\hline Age (weeks) & Males & Females & Total \\
\hline $9-12$ & - & 1 & 1 \\
$13-16$ & 13 & 2 & 15 \\
$17-20$ & 7 & 4 & 11 \\
$21-24$ & 2 & 5 & 7 \\
Total & 22 & 12 & 34 \\
\hline
\end{tabular}

\section{Results}

AQP2 expression is present in the studied periods, and displays a specific pattern: cytoplasmic and in the apical membrane of epithelial cells of collecting tubules and connecting tubules.

Between weeks 9 to 12 , expression in the collecting tubules is more enhanced than in the apical membranes. (Fig. 1A). In the following period (weeks 13 to 20), apical membrane expression is enhanced and doubles cytoplasmic expression, (Fig. 1B-C) which is also more enhanced toward the end of the studied period (Fig. 1D).

In case of connecting tubules, AQP2 expression is absent during weeks 9 to 12 . Starting with weeks 13 to 16 until the end of our study period, expression stronger in the apical membrane of the distal connecting tubules. (Fig 2.).

\section{Discussions}

Aquaporins are organized into membranes as homotetrameres. They facilitate migration of water through different biological membranes. They create a 5 to 50 -fold increase of water permeability of the cell membrane. Aquaporins play an important role in the maternal-fetal fluid balance through regulation of amniotic fluid flow [6].

AQP2 has an important role in concentrating urine. During its inactive state, it is stored in submembrane vesicles. Upon vasopressin stimulation secondary messenger mechanisms are triggered. This leads to insertion of AQP2 into the apical membrane of epithelial cells of the connecting tubules and collecting tubule $[5,7,10,11]$.

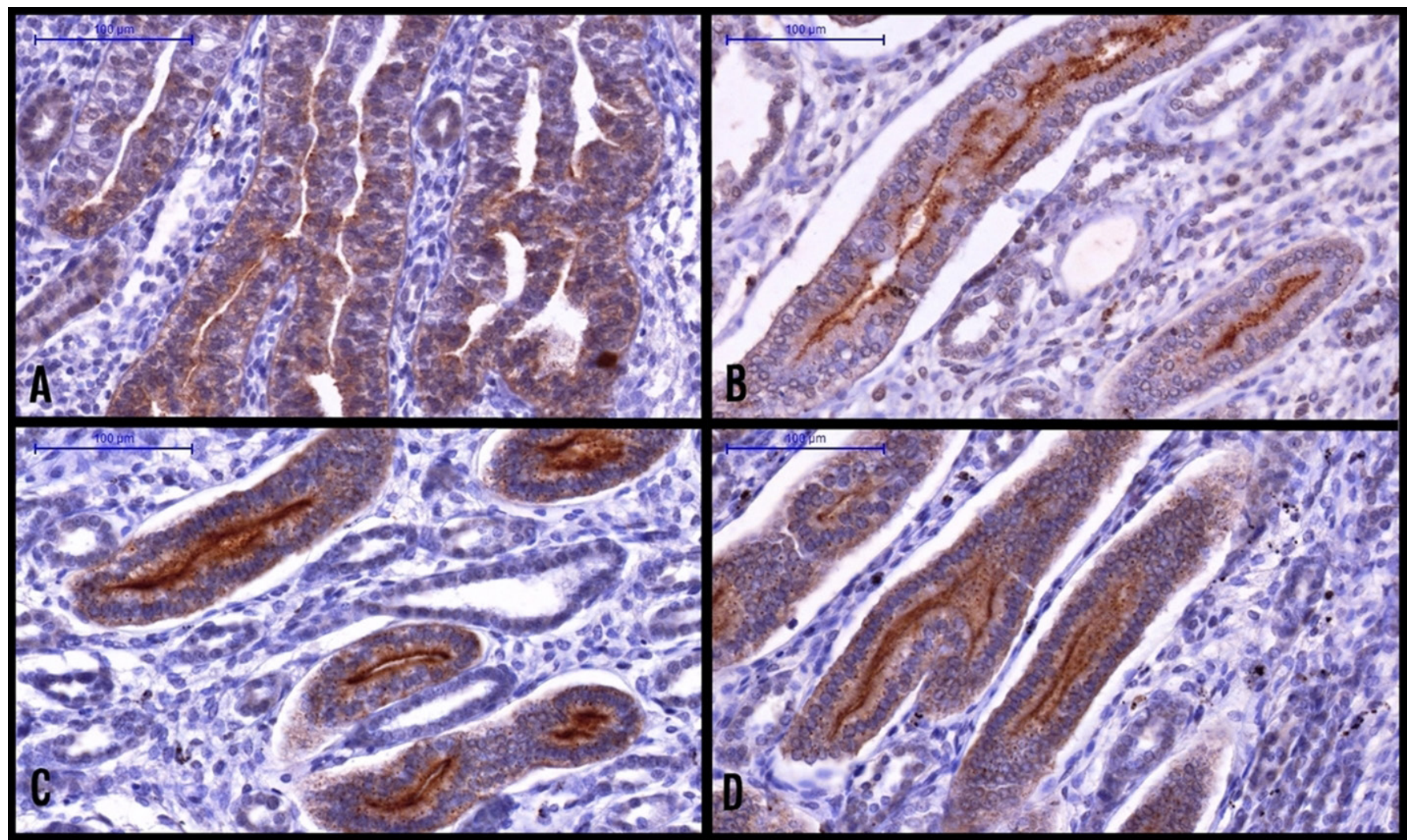

Fig.1. Aquaporin 2 expression in collecting tubules, fetal kidney: A, 9-12 weeks; B, 13-16 weeks; C, 17-20 weeks; D, 21-24 weeks, 20x 


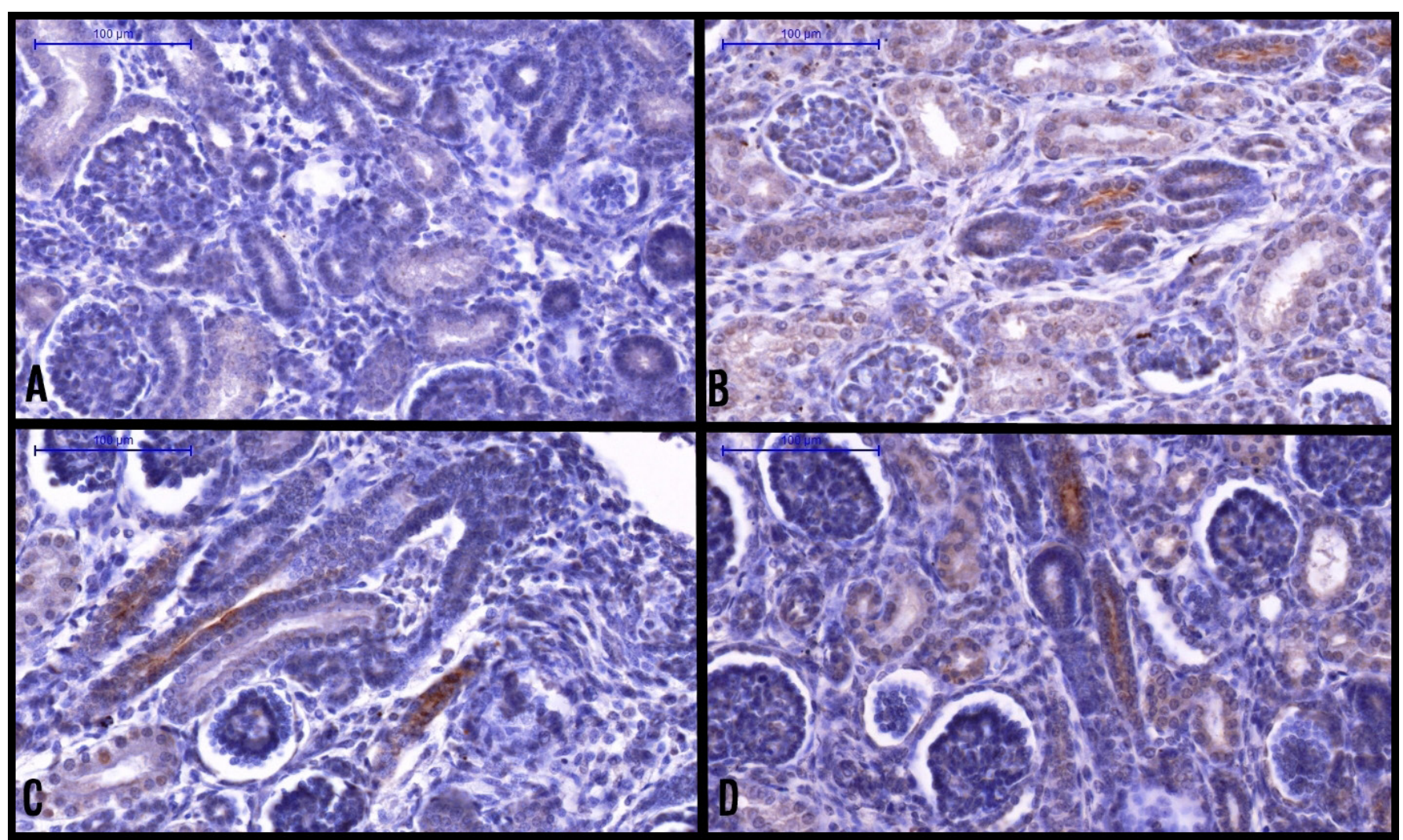

Fig. 2. Aquaporin 2 expression in connecting tubules, fetal kidney: A, 9-12 weeks; B, 13-16 weeks; C, 17-20 weeks; D, 21-24 weeks, 20x

Patients with AQP2 gene mutations show deficits in the ability to concentrate urine and polyuria. In case of rodents, mice with knock-out AQP2 die in their first days of life [12].

According to other studies, AQP2 is expressed in the apical membrane of the ureteric bud as early as the first phases of nephrogenesis (week 12). In the collecting tubule system it was also described in week 12 , and later over the entire course of development [13].

In this study, the earliest AQP2 expression was observed during weeks 9 to 12 in the collecting tubule, but expression was stronger in the cytoplasm than in the apical membrane. Apical membrane expression became more enhanced between weeks 13 to 16 .

Several studies described AQP2 expression as more enhanced in the apical membrane and submembrane vesicles of collecting tubules, and less enhanced in connecting tubules, especially in their distal segments. It was also described in the basal-lateral aspect of the cell membrane [11, $12,14]$.

In this study the expression of AQP2 was identified in the connecting tubules as well, especially in the distal connecting tubules. Its location was confined to the apical membrane. Expression was observed only starting from weeks 13 to 16.

In rats, $\mathrm{AQP} 2$ is present from fetal Day 18 , and displays low levels at birth. In sheep, AQP2 levels at the beginning of the last gestational semester are $17 \%$ of adult levels, and $40 \%$ of adult levels at birth [5]. In this study AQP2 expression was identified during weeks 9 to 12 of fetal life.
According to published reports, the fetal human kidneys shows low levels of AQP2 expression in the second part of gestation, and preterm infants produce diluted urine for several weeks [5].

Ther results of this study suggest a variation of aquaporin stimulation over the time period we studied: initially, expression is more cytoplasmic, and later apical membrane expression becomes enhanced as well. At the end both localizations are stronger. Low AQP2 levels allow for production of large quantities of hypotonic urine, which is essential for maintenance of adequate volumes of aminotic fluid [5].

\section{Conclusions}

AQP2 is essential in the fetal kidney for regulation of amniotic fluid over the course of pregnancy. At the beginning of the fetal period AQP2 protein is present in the cytoplasm of epithelial cells of the collecting tubules and distal connecting tubules. During the fetal period, AQP2 expression in collecting tubules becomes more enhanced in the apical membrane of the cells.

\section{Conflict of interest}

None to declare.

\section{References}

1. Ruchelli ED, Huff SD - Kidney, in Ernst LM, Ruchelli ED, Huff SD (eds): Color Atlas of Fetal and Neonatal Histology. New York. Springer, 2011, 105-116

2. Horster MF, Braun GS, Huber SM - Embryonic renal epithelia: induction, nephrogenesis, and cell differentiation. Physiol Rev. 1999; 79:1157-91. 
3. Mari C, Winyard P - Concise Review: Understanding the Renal Progenitor Cell Niche In Vivo to Recapitulate Nephrogenesis In Vitro. Stem Cells Transl Med. 2015; 4:1463-71.

4. El-Dahr S, Hilliard S, Saifudeen Z - Regulation of kidney development by the Mdm2/Mdm4-p53 axis. J Mol Cell Biol. 2017; 9:26-33.

5. Liu H, Wintour EM - Aquaporins in development - a review. Reprod Biol Endocrinol. 2005;3:18.

6. Hua Y, Jiang W, Zhang W et al - Expression and significance of aquaporins during pregnancy. Front Biosci (Landmark Ed). 2013;18:1373-83.

7. Jung $\mathrm{HJ}$, Kwon $\mathrm{TH}$ - Molecular mechanisms regulating aquaporin-2 in kidney collecting duct. Am J Physiol Renal Physiol. 2016; 311:1318F1328.

8. Takata K, Matsuzaki T, Tajika Y et al - Localization and trafficking of aquaporin 2 in the kidney. Histochem Cell Biol. 2008; 130:197-209.
9. S. K. Agarwal, A. Gupta - Aquaporins: The renal water channels. Indian J Nephrol. 2008; 18: 95-100.

10. Agre P - Homer W. Smith award lecture. Aquaporin water channels in kidney. J Am Soc Nephrol. 2000;11:764-77

11. Kwon TH, Frøkiær J, Nielsen S - Regulation of aquaporin-2 in the kidney: A molecular mechanism of body-water homeostasis. Kidney Res Clin Pract. 2013; 32:96-102.

12. Zhang XY, Wang B, Guan YF - Nuclear Receptor Regulation of Aquaporin-2 in the Kidney. Int J Mol Sci. 2016;17:1105.

13. Devuyst $\mathrm{O}$, Burrow $\mathrm{CR}$, Smith $\mathrm{BL}$ et al - Expression of aquaporins-1 and -2 during nephrogenesis and in autosomal dominant polycystic kidney disease. Am J Physiol. 1996; 271:169-83.

14. Bedford JJ, Leader JP, Walker RJ - Aquaporin expression in normal human kidney and in renal disease. J Am Soc Nephrol. 2003;14:2581-7. 